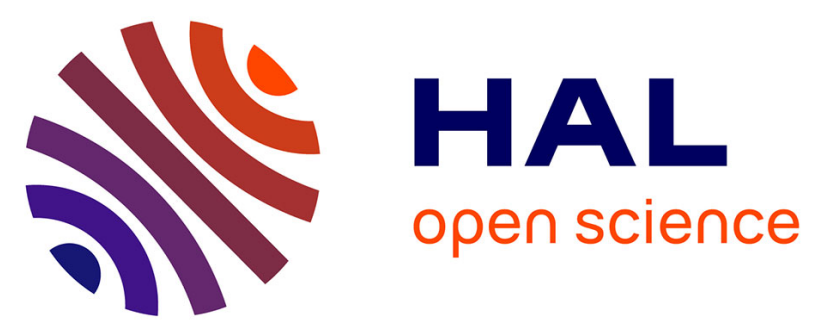

\title{
A Double-Blind Randomized Placebo-Controlled Clinical Trial of Squalamine Ointment for tinea capitis Treatment
}

Oumar Coulibaly, Mahamadou Ali Thera, Abdoulaye Koné, Goita Siaka, Pierre Traoré, Abdoulaye Djimde, Jean-Michel Brunel, Jean Gaudart, Renaud Piarroux, Ogobara K Doumbo, et al.

\section{To cite this version:}

Oumar Coulibaly, Mahamadou Ali Thera, Abdoulaye Koné, Goita Siaka, Pierre Traoré, et al.. A Double-Blind Randomized Placebo-Controlled Clinical Trial of Squalamine Ointment for tinea capitis Treatment. Mycopathologia, 2015, 10.1007/s11046-014-9849-y . hal-01307148

\section{HAL Id: hal-01307148 \\ https://hal-amu.archives-ouvertes.fr/hal-01307148}

Submitted on 26 Apr 2016

HAL is a multi-disciplinary open access archive for the deposit and dissemination of scientific research documents, whether they are published or not. The documents may come from teaching and research institutions in France or abroad, or from public or private research centers.
L'archive ouverte pluridisciplinaire HAL, est destinée au dépôt et à la diffusion de documents scientifiques de niveau recherche, publiés ou non, émanant des établissements d'enseignement et de recherche français ou étrangers, des laboratoires publics ou privés.

\section{(2)(1) $\$$}

Distributed under a Creative Commons Attribution - NonCommercial - NoDerivatives 44.0 


\title{
A Double-Blind Randomized Placebo-Controlled Clinical Trial of Squalamine Ointment for tinea capitis Treatment
}

\author{
Oumar Coulibaly • Mahamadou A. Thera • Abdoulaye K. Koné • Goïta Siaka • \\ Pierre Traoré · Abdoulaye A. Djimdé · Jean-Michel Brunel · Jean Gaudart • \\ Renaud Piarroux $\cdot$ Ogobara K. Doumbo $\cdot$ Stéphane Ranque
}

Received: 25 June 2014/ Accepted: 11 December 2014/Published online: 17 December 2014

(C) Springer Science+Business Media Dordrecht 2014

\begin{abstract}
Background Novel treatments against for tinea capitis are needed, and the natural aminosterol squalamine is a potential topical antidermatophyte drug candidate.

Objectives This phase II randomized double-blind placebo-controlled clinical trial aimed at testing the efficacy and safety of a three-week squalamine ointment regimen for the treatment of tinea capitis.

Patients Males aged 6-15 years presenting with tinea capitis were treated with either topical squalamine ointment or placebo for 3 weeks. The primary endpoint was complete clinical cure. The secondary
\end{abstract}

Trial registration: www.pactr.orgPACTR201306000522355.

O. Coulibaly $\cdot$ R. Piarroux $\cdot$ S. Ranque

IP-TPT UMR MD3, Aix-Marseille Université,

13885 Marseille, France

O. Coulibaly · M. A. Thera · A. K. Koné .

G. Siaka · A. A. Djimdé · O. K. Doumbo

DEAP/MRTC, Faculté de Médecine, Université de

Bamako, Bamako, Mali

P. Traoré

Department of Dermatology, Faculty of Medicine, CNAM

Ex Institut Marchoux, University of Bamako,

PO Box 251 Bamako, Mali

J.-M. Brunel

Centre de Recherche en Cancérologie de Marseille (CRCM), CNRS, UMR7258, Institut Paoli-Calmettes, Marseille, France endpoints were the occurrence of local and/or systemic adverse events, mycological cure, and partial clinical response. Prospective follow-up of clinical adverse events was performed daily.

Results Five patients were treated with $1 \%$ squalamine ointment and 15 with placebo. No complete cure was observed. No clinical or biological adverse event was recorded. A significantly $(p=0.03)$ better hair-growth score, indicating a partial clinical improvement of the tinea capitis lesion, was observed in the patients treated with squalamine compared to those treated with placebo.

Conclusion This three-week squalamine ointment regimen was well tolerated and showed an encouraging partial clinical activity for the treatment of tinea

\author{
J.-M. Brunel \\ UM 105; Inserm, U1068, Aix-Marseille Université, \\ 13009 Marseille, France \\ J. Gaudart \\ UMR912 (SESSTIM), INSERM/AMU/IRD, Aix- \\ Marseille Université, Marseille, France \\ R. Piarroux $\cdot$ S. Ranque \\ Parasitology and Mycology, APHM, CHU Timone- \\ Adultes, 13005 Marseille, France \\ S. Ranque $(\square)$ \\ Laboratoire de Parasitologie Mycologie, CHU Timone, \\ 264 rue Saint Pierre, 13385 Marseille Cedex 5, France \\ e-mail: stephane.ranque@ap-hm.fr
}


capitis. Further studies are needed to evaluate the efficacy of topical squalamine alone against tinea corporis or in combination with a systemic antidermatophyte drug against tinea capitis.

Keywords Aminosterols - Controlled clinical trial · Phase II clinical trial - Topical treatment .

Dermatophytes $\cdot$ Dermatophytoses

\section{Introduction}

Tinea capitis is a common fungal infection of the scalp, hair follicles, and hair shafts [1]. Although it can affect any age group, it is particularly common in school-aged children. It is almost exclusively a disease of childhood, and current evidence suggests that it occurs more often in children of African or Caribbean extraction [2]. In the USA, tinea capitis is the most common dermatophyte infection of childhood, with $90 \%$ of infections caused by the anthropophilic fungi Trichophyton tonsurans [3, 4]. A recent survey carried in Mali, a country in Western Africa, found a high prevalence rate of $55 \%$ of tinea capitis in children attending at primary school in a suburbs of Bamako (the capital city of Mali), with a predominance of anthropophilic dermatophytes [5].

Treatment of tinea capitis is inherently difficult and protracted. Current treatment options included oral and topical drugs with oral therapies providing better outcomes. Griseofulvin remains the only licensed treatment for children in the UK. Nonetheless, many dermatology units in the UK use terbinafine as firstline treatment because it is highly effective, well tolerated, and adherence to treatment is high [2]. However, in developing countries, the cost of these treatments remains very high. In this context, developing an effective and affordable topical therapeutic seems necessary. In recent years, several reports have described the antifungal activity of natural products [6]; among them squalamine was a good drug candidate [7, 8]. Squalamine is a natural aminosterol, first isolated from the tissues of the dogfish shark Squalus acanthias, which possesses numerous therapeutic values including antiangiogenic properties [9]. The aim of this study was to evaluate the efficacy of topical administration of squalamine compared with placebo in the treatment of tinea capitis in children.
Secondary objectives were to assess the safety and to estimate the relative efficacy of squalamine against placebo.

\section{Patients and Methods}

\section{Study Design}

This was a randomized double-blind placebo-controlled clinical trial conducted in primary school in Sirakoro-Meguetana, a semi-urban community located at about $4 \mathrm{~km}$ of Bamako. The trial was registered under the PACTR201306000522355 identification number in the Pan African Clinical Trial Registry (www.pactr.org). The trial included an approximate 2-week screening period and 3-week treatment period. Patients were randomized to receive one of two possible treatments: squalamine (petroleum jelly with $1 \%$ squalamine ointment) or placebo (petroleum jelly ointment). Treatment evaluated and placebo were applied once daily on the lesions for 3 weeks. The investigator, wearing gloves, first roughly cleaned the scalp and then applied the ointment using his finger. Patients were followed up until 4 weeks after the first dose. Patients were to receive their medication once a day, preferably in the morning. A thin layer of ointment (either squalamine or placebo) was applied topically by the investigator.

\section{Study Patients}

The study protocol was approved by the Ethical Committee of Faculty of Medicine and Dentistry of the University of Sciences Techniques and Technologies of Bamako ( $\left.\mathrm{N}^{\circ} 10-82 / \mathrm{FMPOS}\right)$ and was carried out in accordance with the Good Clinical Practice standards. Written informed consent was obtained from each patient and their parents before enrollment. Eligible patients aged 6-15 years had a clinical mycological diagnosis of tinea capitis. Patients underwent complete clinical examination, collection of material from lesions of tinea capitis for mycological examination, and blood samples for biochemical and hematological tests.

Inclusion criteria were male children, aged from 6 to 15 years, attending the primary school of SirakoroMeguetana, with both clinical and mycological 
diagnosis of tinea capitis, normal liver and renal function tests, and no history of allergy.

Non-inclusion criteria were children with dermatophyte infection of the skin and/or who received systemic antifungal therapy within 3 months before inclusion or topical antifungal therapy within 1 month before inclusion. Patients were required to shave their heads at day 0 . Because shaving of head is usual practice for boys, but not for girls in this population, girls were not included to avoid stigmatisation.

\section{Randomization and Blinding}

The blinding and randomization process was as follows: JMB added $1 \%$ squalamine to five out of 20 randomly selected tubes containing $20 \mathrm{~g}$ of petrolatum-based ointment. The tubes were anonymized and identified with a unique number from 1 to 20. Only JMB knew the randomization key; he was neither involved in the drug administration nor in the clinical monitoring of patients. Twenty patients received daily topical application (either squalamine or placebo) during 3 weeks. The treatment evaluated and placebo had the same visual appearance. Neither the clinician in charge of the patient's treatment follow-up nor the patient knew the exact nature of the administered treatment. The unblinding occurred after the "freezing" of the database before the statistical analysis.

\section{Patient's Follow-up}

At day 0 , the patients were clinically examined by qualified physicians, and dermatophytes lesions were characterized. Blood samples were collected to evaluate kidney and liver functions and blood cell count. Wood's light examination was performed in a darkened room to detect ectothrix hair invasion caused by Microsporum spp. Samples were collected from dermatophytoses lesions. Tinea capitis lesions were collected by scraping with a sterile curette and a sterile compress. In areas of alopecia, scales and broken hair were collected and used for direct examination. A second sample collected directly with the sterile compress was kept at room temperature in sealed plastic bags and then transported to the ParasitologyMycology Laboratory at the University Hospital of Marseille where samples were inoculated onto Sabouraud cycloheximide agar medium (bioMérieux, France). Cultures were maintained for 6 weeks for positive culture identification. Positive cultures were examined macroscopically and microscopically for species identification on the basis of cultural characteristics, pigment production, and microscopic examination in chloral-lactophenol cotton blue preparation. Treatment was started after shaving of the patients' skull and was administered once daily. Clinical efficacy and adverse events were also monitored daily.

At day 7, samples were collected from tinea capitis for mycological examination. Blood sample was collected for laboratory tests. Finally, administration of treatment was continued in the absence of cure or major adverse effects.

At day 21, the samples were collected from lesions of tinea capitis. Blood samples were also collected to monitor kidney and liver functions and blood cell counts. Treatment was ended. The patients with residual tinea capitis were then treated with $20 \mathrm{mg} /$ $\mathrm{kg} / \mathrm{d}$ oral griseofulvin for 6-8 weeks.

\section{Efficacy Evaluations}

At each study visit, signs and symptoms of tinea capitis were assessed and samples of tinea capitis lesions were collected by the investigator for mycological analysis, as detailed above. The primary efficacy endpoint was a complete clinical cure at week 3. The first secondary efficacy endpoint was mycological cure at week 3. Complete cure was defined as negative mycology (negative culture) and clinical cure (no clinical symptoms of tinea capitis, and normal hair growth). Mycological cure was defined as negative culture. The second secondary efficacy endpoint was partial clinical response, which was quantified using a hair-growth score and the diameter of lesions. The diameter of lesions was measured using a sliding caliper gauge. Hair growth was assessed clinically using a score of 0 (no growth) to 5 (uniform growth) in comparison with healthy areas of the patient's scalp.

\section{Safety Evaluations}

Adverse events were assessed by the investigators at each visit. Safety variables assessed included vital signs measurements, physical examination, and laboratory test results. There was a particular focus on aspartate aminotransferase (AST), alanine aminotransferase (ALT), alkaline phosphatase and total 
bilirubin, and hematological parameters (white blood cells count, packed cell volume, and platelet counts).

\section{Susceptibility Testing}

In vitro susceptibility testing of squalamine against the patients' isolates was performed as described in [6] using the M38-A2 micro-broth dilution protocol of the Clinical and Laboratory Standards Institute.

\section{Statistical Analysis}

Patients were randomized in a 1:3 ratio in the group treated with squalamine or in the placebo group. To test the difference between $2 \%$ cure rate in placebo group and $80 \%$ cure rate in the squalamine group, with a $5 \%$ type I error and $90 \%$ power, number needed to treat was 20 patients: five in the squalamine group and 15 in the placebo group. All data were collected onto case report forms that were kept confidential and checked daily by a study supervisor. Data were entered into a MS ACCESS ${ }^{\mathrm{TM}}$ database and checked by the supervisor. The data were presented as mean ( \pm standard deviation) for quantitative variables and percentages for qualitative variables. The primary endpoint was complete cure. The secondary endpoints were safety, mycological cure (negative mycology), and partial clinical response (hair-growth score and/or reduction in the diameters of lesion). The proportions of patients with an endpoint in either squalamine or placebo treatment groups were compared using the Fisher's exact test. The same analysis was performed considering each lesion of tinea capitis, using the generalized estimating equations option to account for the non-independence of multiple tinea capitis lesions in a given patient. Statistical analyses were performed using SAS, version 9.2 (SAS Institute, Cary, NC). All tests were two-sided with a $p<0.05$ significance level.

\section{Results}

The study was conducted in May 2011. Twenty male patients aged 6-15 years were included. Five were treated with topical squalamine and 15 with placebo. The data at days 0,7 , and 21 of both squalamine and placebo groups are tabulated in Table 1 . There were no lost to follow-up. The patients' baseline characteristics were homogenous among treatment groups (Table 1). At day 0 , there were 10 and 27 lesions of tinea capitis, mean number of lesions per child $2 \pm 1.2$ and $1.8 \pm 0.8(p>0.05)$, in the squalamine and placebo groups, respectively.

Microsporum audouinii was the predominant pathogen in this study. The MIC of terbinafine was $<0.25 \mathrm{mg} / \mathrm{l}$ in all 21 isolates. All patients in the squalamine group were infected by $M$. audouinii. The means of MICs of squalamine were $11.2 \pm 4.4$ and $12.4 \pm 4.2(p=0.38)$ for $M$. audouinii isolated in patients in the squalamine and the placebo groups, respectively.

At day 7 (Table 1), mean diameters of lesions were $4.9 \pm 4$ and $4 \pm 2.7(p=0.78)$ in the squalamine and placebo groups, respectively. The hair-growth scores in each group did not differ $(p=1.00)$. Neither complete nor mycological cure occurred in any patient in this study.

At day 21 (Table 1), mean diameters of lesions were $3.9 \pm 3.2$ versus $4 \pm 2.5(p=0.92)$ in the squalamine and placebo groups, respectively. As shown in Fig. 1, the mean hair-growth score was significantly higher $(2.1 \pm 8.9$ vs. $1.1 \pm 0.6, p=0.039)$ in the squalamine group than in the placebo group. A score $>1$ for hair growth occurred in $70 \%$ [95 \% CI (0.35-0.93)] versus $18.5 \%$ [95\% CI $(0.06-0.38), p=0.005$ ] in lesions of tinea capitis in the squalamine and placebo groups, respectively. Complete cure was not recorded. However, a negative mycological culture of four tinea capitis lesions was observed in two $(10 \%)$ patients in the placebo group $(p=1.00)$. With respect to safety, no clinical or biological adverse event was prospectively recorded during the entire study.

\section{Discussion}

In this study, although no complete tinea capitis cure occurred in the patients who were treated with topical squalamine, a statistically significantly higher hairgrowth score within the tinea capitis lesions, indicative of a partial clinical response, was observed in the patients treated with squalamine. In fact, the currently available topical treatment is effective against tinea capitis only when combined with a systemic antidermatophyte drug, most probably because the dermatophytes are located within the hair [2]. Although systemic therapy, such as oral griseofulvin, effectively penetrates the infected hair shaft, topical shampoos, 
Table 1 Clinical and mycological characteristics at baseline and at day 7 and day 21 of follow-up of the children participating to this double-blind randomized placebo-controlled clinical trial of squalamine ointment for tinea capitis treatment

\begin{tabular}{|c|c|c|c|c|c|c|c|c|c|}
\hline & \multicolumn{3}{|l|}{ Day 0} & \multicolumn{3}{|l|}{ Day 7} & \multicolumn{3}{|l|}{ Day 21} \\
\hline & Squalamine & Placebo & $p$ & Squalamine & Placebo & $p$ & Squalamine & Placebo & $p$ \\
\hline$N$ & 5 & 15 & - & 5 & 15 & - & 5 & 15 & - \\
\hline \multicolumn{10}{|l|}{ Clinical evaluation } \\
\hline \multicolumn{10}{|c|}{ Number of tinea capitis lesions } \\
\hline Total & 10 & 27 & - & 10 & 27 & - & 10 & 27 & - \\
\hline Mean per patient $( \pm \mathrm{SD})$ & $2( \pm 1.2)$ & $1.8( \pm 0.8)$ & 0.67 & $2( \pm 1.2)$ & $1.8( \pm 0.8)$ & 0.67 & $2( \pm 1.2)$ & $1.8( \pm 0.8)$ & 0.67 \\
\hline \multicolumn{10}{|l|}{ Diameter of the lesions } \\
\hline Mean $( \pm \mathrm{SD})$ & $5.1( \pm 3.9)$ & $4.9( \pm 2.6)$ & 0.88 & $4.9( \pm 4.0)$ & $4.6( \pm 2.7)$ & 0.78 & $3.9( \pm 3.2)$ & 4. $( \pm 2.5)$ & 0.92 \\
\hline \multicolumn{10}{|l|}{ Hair growth } \\
\hline Mean score $( \pm \mathrm{SD})$ & - & - & - & $1.2 \pm 0.4$ & $1.0( \pm 0.2)$ & 1.00 & $2.1( \pm 8.9)$ & $1.1( \pm 0.6)$ & 0.039 \\
\hline \multicolumn{10}{|l|}{ Mycological evaluation } \\
\hline $\begin{array}{l}\text { Positive dermatophytes } \\
\text { cultures }\end{array}$ & $100 \%$ & $100 \%$ & & $100 \%$ & $100 \%$ & & $100 \%$ & $86.7 \%$ & 1.00 \\
\hline \multicolumn{10}{|l|}{ Dermatophyte species } \\
\hline Trichophyton soudanense & 0 & $26.5 \%$ & & $0 \%$ & $33 \%$ & & $0 \%$ & $23 \%$ & - \\
\hline Microsporum audouinii & $100 \%$ & $66.5 \%$ & & $100 \%$ & $60.0 \%$ & & $100 \%$ & $69.3 \%$ & - \\
\hline $\begin{array}{l}\text { T. soudanense } \\
\text { and M. audouinii }\end{array}$ & 0 & $7.0 \%$ & & 0 & $7.0 \%$ & & 0 & $7.7 \%$ & - \\
\hline
\end{tabular}

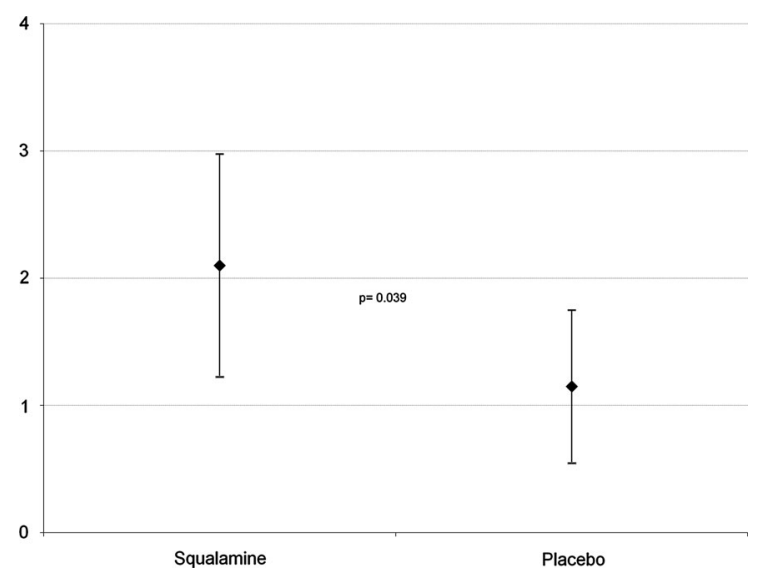

Fig. 1 Mean $( \pm S D)$ hair-growth score in the tinea capitis lesions treated for 3 weeks with squalamine ointment $(n=10$ patients) or placebo ( $n=27$ patients)

including ketoconazole and selenium sulfide, are recommended as adjunctive therapeutic options and to prevent spread of infection [10-12]. This partial efficacy of topical squalamine against dermatophytes was in keeping with recent experimental data on the use of topical squalamine treatment for Staphylococcus aureus skin decolonization in mice [13]. In this present study, a negative dermatophyte culture occurred in two patients with stable clinical lesions in the placebo group. The most probable explanation is a false negative culture results due to relatively low sensitivity of culture for the diagnosis of dermatophytosis [14], mainly because of the difficulties in obtaining good quality samples for dermatophyte culture.

However, this study showed a favorable safety profile of squalamine when administered topically as no clinical or biological adverse event was recorded in the treated patients. Yet, this finding should be interpreted taking into account the small number of patients treated. The favorable safety index of topical squalamine was in line with the recent findings of the absence of genotoxicity and its low cytotoxic activity against $\mathrm{CHO}$ cells suggesting a low cytogenetic impact on eukaryotic cells [15]. A phase II study demonstrated that this aminosterol compound could be safely administered to cancer patients in a five-day continuous infusion regimen [16]. Moreover, squalamine well tolerated without any major side effects at doses up to $250 \mathrm{mg} /$ day in adults included in phase III trials for the treatment of age-related macular degeneration and prostate cancer [13]. Although no pharmacokinetic data are available, it is likely that squalamine plasma levels when administered topically 
are much lower than those obtained when administered intravenously, thus suggesting that a squalamine ointment could be safe for topical use. Aminosterols also possess interesting in vitro antibacterial and antifungal activities, especially squalamine minimum inhibitory concentrations (MICs) ranged from 4 to $16 \mathrm{mg} / \mathrm{l}$ against various clinical dermatophytes species $[7,8]$. Thus, using a $1 \%$ squalamine-formulated petrolatum-based cream ointment, as in the present study, the local squalamine concentration would exceed more than 1,000 times the dermatophyte MIC, suggesting a good microbiological efficiency for a topical use [13]. With respect to the in vitro activity of aminosterols against dermatophytes, we recently showed that another aminosterol derivative had lower MICs than squalamine [7] and might have an enhanced activity against dermatophytes.

There were three major limitation of this phase II clinical trial. First, the children who were randomized in the squalamine group were infected by the single M. audouinii species. Thus, this study's findings can only cautiously be extrapolated to tinea capitis caused by other dermatophytes, although in vitro Trichophyton soudanense was at least as susceptible to squalamine as $M$. audouinii. Second, squalamine ointment was administered once in a day, which could have been insufficient, regarding the inefficacy of the available antidermatophyte topical treatments alone against treat tinea capitis to date [2]. Currently, the recommended therapy for tinea capitis in children is a 6- to 8-week course of oral griseofulvin [17]. This study's objective to cure $80 \%$ of the children in 3 weeks can retrospectively be regarded as too optimistic and even unrealistic. Nonetheless, considering the randomized double-blind design, the partial clinical response evidenced in this study should be considered as a very encouraging result. In 2007, a Cochrane review of systemic antifungal treatment for tinea capitis in children concluded that newer treatments, including terbinafine, have equivalent efficacy and safety profiles to griseofulvin and allow shorter treatment [2]. Lipozencic et al. [18], in a double-blind randomized trial in children with tinea capitis due to Microsporum spp, concluded that terbinafine therapy for 6 weeks could represent an alternative to griseofulvin for the treatment of Microsporum tinea capitis. The third limitation was that the dose of squalamine applied daily on the lesions was not quantified. A number of antifungal drugs with proven efficacy are available for the treatment of tinea capitis. However, varying dosage schedules, changes in epidemiology, and increasing drug resistance are factors that might hamper treatment's efficacy [19]. The partial clinical response found in this phase II study suggests that topical squalamine would be active against tinea corporis, which is usually treated with topical antifungals alone or against tinea capitis in combination with a systemic antifungal. In our setting, systemic antidermatophyte agent such griseofulvin should be preferred because of its relatively lower cost and the high prevalence of Microsporum sp. in this population.

\section{Conclusion}

This phase II study evidenced the safety of topical squalamine treatment and a partial clinical efficacy of a three-week regimen for the treatment of tinea capitis lesions. This encouraging result supports further studies, which should aim at evaluating the efficacy of various doses of squalamine ointments as monotherapy against tinea corporis or in combination with a systemic antifungal like griseofulvin against tinea capitis. In both situations, squalamine has the potential to shorten dermatophytosis treatment duration with subsequent improvement of compliance to treatment schedules and costs reduction.

Acknowledgments We would like to thank the school principal Jean-André Douyon for his help.

Conflict of interest None to declare.

\section{References}

1. Mohrenschlager M, Seidl HP, Ring J, Abeck D. Pediatric tinea capitis: recognition and management. Am J Clin Dermatol. 2005;6:203-13.

2. Blaithin M, Roderick H, Racheal M. The diagnosis and management of tinea. BMJ. 2012;10(345):e4380. doi:10. 1136/bmj.e4380.

3. Sharma V, Hall JC, Knapp JF, Sarai S, Galloway D, Babel DE. Scalp colonization by Trichophyton tonsurans in an urban pediatric clinic. Asymptomatic carrier state? Arch Dermatol. 1988;124:1511-3.

4. Abdel-Rahman SM, Farrand N, Schuenemann E, Stering TK, Preuett B, Magie R, Campbell A. The prevalence of infections with Trichophyton tonsurans in schoolchildren: the CAPITIS study. Pediatrics. 2010;125:966-73. 
5. Coulibaly O, Thera MA, Djimde AA, Ranque S. Dermatomycoses in rural and peri-urban schoolchildren in Mali. Mycoses. 2011;54:68.

6. Sanguinettti M, Posteraro B, Romano L, Battaglia F, Lopizzo T, De Carolis E, Fadda G. In vitro activity of Citrus bergamia (bergamot) oil against clinical isolates of dermatophytes. J Antimicrob Chemother. 2007;59:305-8.

7. Coulibaly O, Alhanout K, L'Ollivier C, Brunel JM, Thera MA, Djimdé AA, Doumbo OK, Piarroux R, Ranque S. In vitro activity of aminosterols against dermatophytes. Med Mycol. 2013;51:309-12.

8. Alhanout K, Brunel JM, Ranque S, Rolain JM. In vitro antifungal activity of aminosterols against mould isolates from cystic fibrosis patients. J Antimicrob Chemother. 2010;65:1307-9.

9. Brunel JM, Salmi C, Loncle C, Vidal N, Letourneux Y. Squalamine: a polyvalent drug of the future? Curr Cancer Drug Targets. 2005;5:267-72.

10. Chan YC, Friedlander SF. New treatments for tinea capitis. Curr Opin Infect Dis. 2004;17:97-103.

11. Greer DL. Successful treatment of tinea capitis with $2 \%$ ketoconazole shampoo. Int J Dermatol. 2000;39:302-4.

12. Bookstaver PB, Watson HJ, Winters SD, Carlson AL, Schulz RM. Prophylactic ketoconazole shampoo for tinea capitis in a high-risk pediatric population. J Pediatr Pharmacol Ther. 2011;16:199-203.

13. Djouhri-Bouktab L, Alhanout K, Andrieu V, Raoult D, Rolain JM, Brunel JM. Squalamine ointment for Staphylococcus aureus skin decolonization in a mouse model. J Antimicrob Chemother. 2011;66:1306-10.
14. Paugam A, L'ollivier C, Viguié C, Anaya L, Mary C, de Ponfilly G, Ranque S. Comparison of real-time PCR with conventional methods to detect dermatophytes in samples from patients with suspected dermatophytosis. J Microbiol Methods. 2013;95:218-22.

15. Alhanout K, Di Giorgio C, De Méo M, Brunel JM. Nongenotoxic assessment of a natural antimicrobial agent: squalamine. Anti-Infective Agents. 2014;12:75-79.

16. Herbst RS, Hammond LA, Carbone DP, Tran HT, Holroyd KJ, Desai A, Williams JI, Bekele BN, Hait H, Allgood V, Solomon S, Schiller JH. A phase I/IIA trial of continuous five-day infusion of squalamine lactate (MSI-1256F) plus carboplatin and paclitaxel in patients with advanced nonsmall cell lung cancer. Clin Cancer Res. 2003;15:4108-15.

17. Bennett ML, Fleischer AB, Loveless JW, Feldman SR. Oral griseofulvin remains the treatment of choice for tinea capitis in children. Pediatr Dermatol. 2000;17:304-9.

18. Lipozencic J, Skerlev M, Orofino-Costa R, Zaitz VC, Horvath A, Chouela E, Romero G, Gourmala N, Paul C, Tinea Capitis Study Group. A randomized double-blind, parallel group, duration funding study of oral terbinafine and open-label, high-dose griseofulvin in children with tinea capitis due to Microsporum species. Br J Dermatol. 2002;146:816-23.

19. Grover C, Arora P, Manchanda V. Comparative evaluation of griseofulvin, terbinafine, and fluconazole in the treatment of tinea capitis. Int J Dermatol. 2012;51:455-8. 